\title{
Contemporary Management of Aneurysmal Subarachnoid Hemorrhage: A Literature Review
}

\author{
Nikki Yeo ${ }^{1, \#} \quad$ Luke Terrett ${ }^{1, \#} \quad$ Arun K. Gupta ${ }^{2}$ \\ ${ }^{1}$ Neurosciences Critical Care Unit, Addenbrooke's Hospital, \\ Cambridge University Hospitals NHS Foundation Trust, Cambridge, \\ United Kingdom \\ ${ }^{2}$ Department of Anaesthesia and Neurosciences Critical Care Unit, \\ Addenbrooke's Hospital, Cambridge University Hospitals NHS \\ Foundation Trust, Cambridge, United Kingdom \\ J Neuroanaesthesiol Crit Care 2019;6:131-139
}

\begin{abstract}
Address for correspondence Nikki Yeo, MBChB, FCICM, Neurosciences Critical Care Unit, Box 1, Cambridge University Hospitals NHS Foundation Trust, Hills Road, Cambridge CB2 0QQ, United Kingdom (e-mail: nikki.yeo@addenbrookes.nhs.uk).
\end{abstract}

\begin{abstract}
Subarachnoid hemorrhage (SAH) results in significant morbidity and mortality, which leads to additional economic and psychological burden. Atraumatic SAH is normally secondary to a ruptured aneurysm. Diagnosis of aneurysmal subarachnoid hemorrhage (aSAH) is usually made by computed tomography (CT) of the head: noncontrast CT identifies the hemorrhage, and CT angiography identifies the aneurysm.

The management of aSAH is focused on securing the aneurysm and prevention of secondary brain injury. Definitive treatment of the aneurysm is done by endovascular coiling or surgical clipping, which should be performed as soon as possible with avoidance of hypertension and strict blood pressure management prior to aneurysm securement. Routine use of nimodipine is recommended by international guidelines as a neuroprotectant. Common neurological complications of aSAH are hydrocephalus, seizures, vasospasm, and delayed cerebral ischemia (DCl). Detection of $\mathrm{DCl}$, especially in patients with poor-grade aSAH, can be challenging. In addition to clinical examination,

Keywords

- subarachnoid hemorrhage

- management

- aneurysm

- vasospasm

- delayed cerebral ischemia multiple radiological modalities can be used, with digital subtraction angiography being the gold standard but CT perfusion gaining an increasingly important role. Extracranial complications including cardiac dysfunction, pulmonary edema, and electrolyte abnormalities are common, causing significant morbidity and mortality. In this review, a PubMed literature search was conducted using the terms "subarachnoid hemorrhage" and "management," with results limited to past 5 years. Journal articles were hand-selected by the authors based on relevance, and the references were reviewed to identify additional relevant publications. Historically important publications were also included.
\end{abstract}

\section{Introduction}

Subarachnoid hemorrhage (SAH) accounts for 5 to $10 \%$ of strokes in the United States, affecting a younger patient group compared with other forms of stroke. ${ }^{1}$ The overall prognosis for SAH remains poor, with one-third of the patients dying and one-third of survivors living in a dependent state. ${ }^{2}$ This results in significant economic loss and psychological and social burden. ${ }^{3,4}$ Patients who survive continue to experience deficits in cognition, quality of life, and mood disorders and fatigue in the long term. ${ }^{5}$ Increased long-term mortality rate in survivors with good recovery, from cardiovascular and cerebrovascular causes, is also observed. ${ }^{6}$

Eighty percent of atraumatic SAH cases are caused by a ruptured intracranial aneurysm. ${ }^{1}$ Based on a systematic review, the prevalence of intracranial aneurysms in adults is estimated to be 2 to $5 \% .^{7}$ There is considerable variation worldwide, but the overall incidence of aneurysmal subarachnoid hemorrhage (aSAH) is estimated to be 9 per 100,000 population. ${ }^{8}$ Countries with reported high incidence

\#Both the authors contributed equally to this manuscript.

received

February 26, 2019

accepted after revision

March 21, 2019

published online

June 3, 2019
DOI https://doi.org/

10.1055/s-0039-1688898

ISSN 2348-0548.
Copyright @2019 Indian Society of Neuroanaesthesiology and Critical Care
License terms

() (1) $\ominus \circledast$ 
are Finland and Japan, whereas South and Central America have a lower incidence. ${ }^{9}$

Formation of aneurysm commonly occurs at major arterial branch points of the circle of Willis, due to hemodynamic stress and the associated inflammatory response. ${ }^{10}$ This causes degeneration of the internal elastic lamina, resulting in thinning of tunica media. Factors associated with increased risk of intracranial aneurysms are family history (first-degree relatives with intracranial aneurysm) and genetic syndromes (e.g., Ehlers-Danlos syndrome and polycystic kidney disease). ${ }^{1}$

Risks associated with aneurysm rupture can be divided into modifiable and nonmodifiable factors. Modifiable risk factors are smoking, hypertension, alcohol abuse, and sympathomimetic drug use. Nonmodifiable risk factors are female sex, black and Hispanic ethnic groups, aneurysm-related factors: (1) presence of unruptured cerebral aneurysm, (2) larger (> $7 \mathrm{~mm}$ ) aneurysms, and (3) posterior circulation aneurysms. ${ }^{1,9}$

The pathogenesis of SAH is related to the disruption of homeostasis between cerebral blood flow, blood components, and vascular wall architecture. Following SAH, an inflammatory response occurs in the subarachnoid space, causing a cascade of responses: vasoconstriction, meningitis, and edema. ${ }^{11}$ The brain injury component can be divided into the early and delayed phases. The early phase manifests as the neurological status of the patients at the time of bleed, as a result of transient global brain ischemia from raised intracranial pressure and reduced cerebral blood flow. The delayed phase, due to delayed ischemia, is seen in one-third of patients and typically occurs 3 to 14 days after the bleed. ${ }^{12}$

\section{Diagnosis}

The hallmark presenting symptom is "the worst headache of my life" or "thunderclap headache," reported in $80 \%$ of patients who were able to provide history. ${ }^{13}$ In 20 to $40 \%$ of the patients, sentinel headache may occur within 2 to 8 weeks of aSAH. ${ }^{1}$

Noncontrast computed tomography (CT) of the head has high sensitivity (close to $100 \%$ ) within the initial 3 days of symptom onset. In fact, within 6 hours from symptom onset, CT has a sensitivity and specificity of $100 \% .{ }^{14}$ However, by days 5 to 7 , the sensitivity declines to $50 \% .{ }^{15}$ If clinical suspicion of SAH remains high despite a nondiagnostic CT scan, lumbar puncture should be performed to detect xanthochromia in the cerebrospinal fluid (CSF), which can be present as early as 6 hours (and is reliably present after 12 hours).8,9,16

CT angiography (CTA) has high sensitivity (77-97\%) and specificity (87-100\%) for detection of cerebral aneurysms (-Fig. 1). However, the sensitivity decreases with smaller aneurysms ( $<3 \mathrm{~mm}$ ), and if inconclusive, digital subtraction angiography (DSA) is indicated, which remains the gold standard for aneurysm diagnosis and anatomy assessment for treatment ( - Fig. 2) . $^{9,12,17}$

\section{Grading}

The three main clinical grading systems are (1) Hunt and Hess scale, (2) World Federation of Neurological Surgeons (WFNS) grading scale, and (3) Prognosis on Admission of Aneurysmal Subarachnoid Hemorrhage (PAASH) grading scale. ${ }^{18-20}$ These grading criteria are summarized in - Table $\mathbf{1}$.

Both PAASH and WFNS grading scales are objective and are good predictors of prognostic outcomes. ${ }^{21}$ Hunt and Hess scale was originally designed to evaluate the risk of surgical intervention. Although widely used, one of its main limitations is the high interobserver variability due to its subjective grading. ${ }^{6,22}$

Radiological grading systems include Fisher and modified Fisher scales, summarized in -Table 2. The Fisher scale was designed to predict risk of vasospasm, but not clinical outcome. ${ }^{23}$ The modified Fisher scale introduced in 2006 takes into account not only SAH but also the separate and additive risks of intraventricular hemorrhage. It has better predictive power on the risk of vasospasm, with ascending grades associated with increasing risks. ${ }^{24,25}$

\section{Management of the Patient with Unsecured Aneurysm}

Rebleeding prior to securing the aneurysm has a significant impact on outcomes and is associated with a mortality rate of 20 to $60 \% .{ }^{26}$ It occurs in 8 to $23 \%$ of patients with the majority of episodes (50-90\%) occurring extremely early (within 6 hours of the primary event). ${ }^{26,27}$ Although multiple risk factors for rebleeding have been identified, the results have often been conflicting and contradictory, and many of them are nonmodifiable in the acute setting. ${ }^{26-28}$ As a result, much of the focus clinically is on the following treatable factors: (1) elevated blood pressure, (2) clot stabilization (antifibrinolysis), and (3) early definitive aneurysm obliteration.

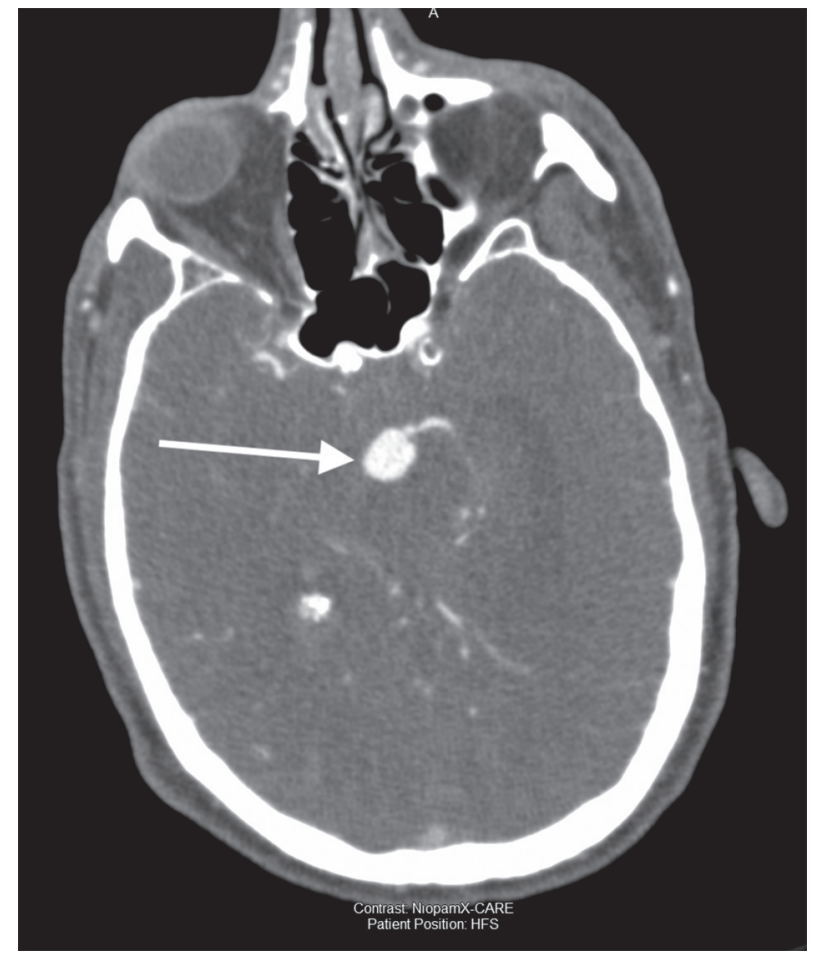

Fig. 1 Computed tomography angiography showing a large basilar aneurysm (white arrow). 

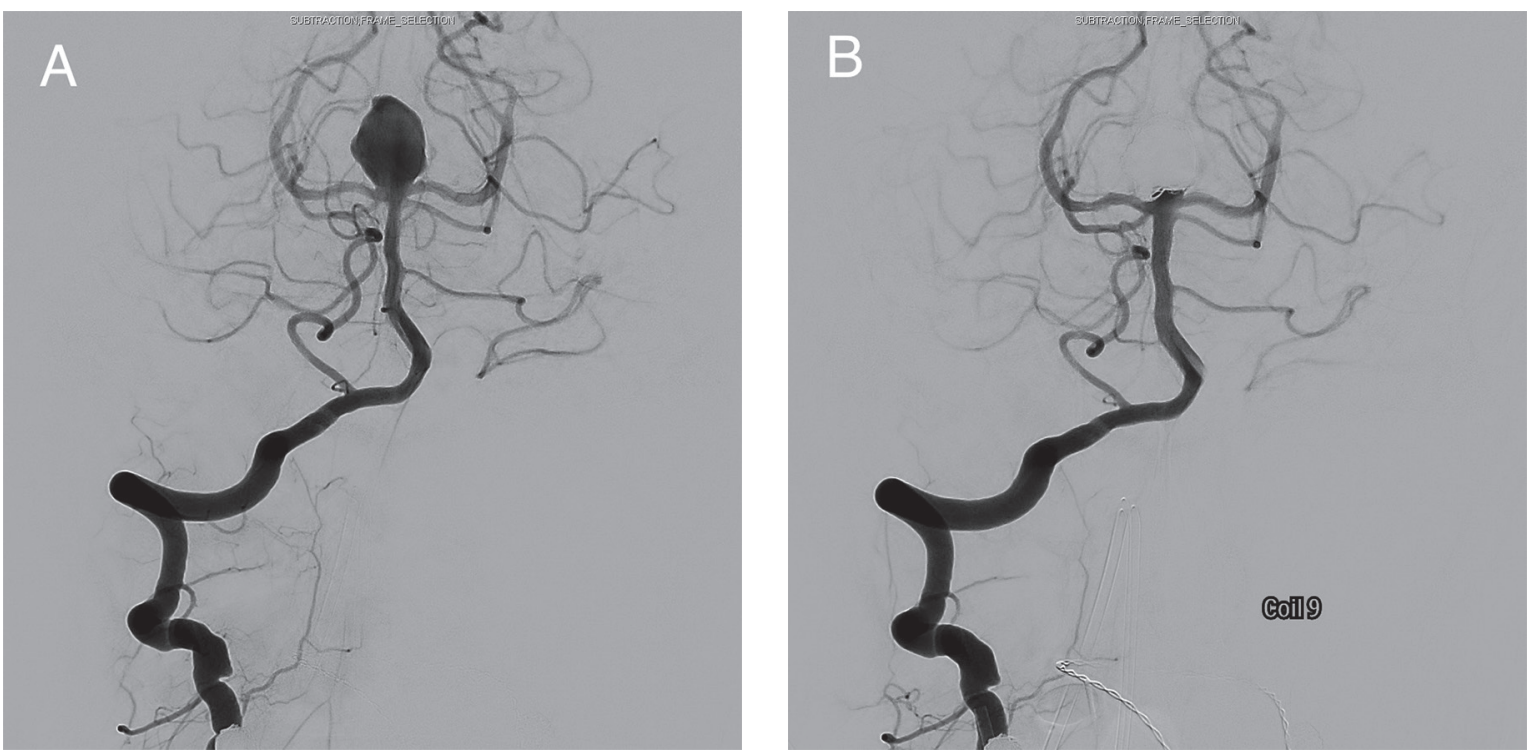

Fig. 2 Digital subtraction angiography. (A) A large basilar aneurysm prior to intervention. The aneurysm is filled with contrast and is readily visible. (B) After coiling. Platinum coils are visible within the aneurysm and the aneurysm itself no longer filled with contrast.

Table 1 Clinical grading scales

\begin{tabular}{|c|c|c|c|}
\hline Grade & PAASH grading scale (1999) ${ }^{18}$ & WFNS grading scale $(1988)^{19}$ & Hunt and Hess scale $(1968)^{20}$ \\
\hline 1 & GCS 15 & GCS 15 & $\begin{array}{l}\text { Asymptomatic or minimal headache } \\
\text { Slight nuchal rigidity }\end{array}$ \\
\hline 2 & GCS 11-14 & $\begin{array}{l}\text { GCS } 13-14 \\
\text { No focal deficits }\end{array}$ & $\begin{array}{l}\text { Moderate to severe headache } \\
\text { Nuchal rigidity } \\
\text { No neurological deficit except cranial nerve palsy }\end{array}$ \\
\hline 3 & GCS $8-10$ & $\begin{array}{l}\text { GCS } 13-14 \\
\text { Focal deficits }\end{array}$ & $\begin{array}{l}\text { Drowsiness, confusion } \\
\text { Mild focal deficit }\end{array}$ \\
\hline 4 & GCS 4-7 & GCS 7-12 & $\begin{array}{l}\text { Stupor } \\
\text { Moderate to severe hemiparesis, possibly early } \\
\text { decerebrate rigidity } \\
\text { Vegetative disturbances }\end{array}$ \\
\hline 5 & GCS 3 & GCS 3-6 & $\begin{array}{l}\text { Deep coma } \\
\text { Decerebrate rigidity } \\
\text { Moribund appearance }\end{array}$ \\
\hline
\end{tabular}

Abbreviations: GCS, Glasgow coma scale; PAASH, Prognosis on Admission of Aneurysmal Subarachnoid Hemorrhage; WFNS, World Federation of Neurological Surgeons.

Table 2 Radiographic grading scales

\begin{tabular}{|l|l|l|}
\hline Grade & Fisher scale $^{23}$ & Modified Fisher scale ${ }^{24}$ \\
\hline 0 & No SAH or IVH & $\begin{array}{l}\text { Minimum or thin SAH, } \\
\text { no IVH in either lateral } \\
\text { ventricle }\end{array}$ \\
\hline 2 & $\begin{array}{l}\text { Diffuse, thin SAH, no } \\
\text { clot }>1 \text { mm in thickness }\end{array}$ & $\begin{array}{l}\text { Minimum or thin SAH } \\
\text { with IVH in both lateral } \\
\text { ventricles }\end{array}$ \\
\hline 3 & $\begin{array}{l}\text { Localized thick layer } \\
\text { of subarachnoid clot }> \\
1 \text { mm in thickness }\end{array}$ & $\begin{array}{l}\text { Thick SAH, no IVH in } \\
\text { either lateral ventricle }\end{array}$ \\
\hline 4 & $\begin{array}{l}\text { Predominant IVH or } \\
\text { intracerebral hemorrhage } \\
\text { without thick SAH }\end{array}$ & $\begin{array}{l}\text { Thick SAH with IVH in } \\
\text { both lateral ventricles }\end{array}$ \\
\hline
\end{tabular}

Abbreviations: IVH, intraventricular hemorrhage; SAH, subarachnoid hemorrhage.

\section{Blood Pressure Management}

Elevated systolic blood pressure on admission is associated with a higher risk of rebleeding. ${ }^{26,27}$ In theory, the "immature" fibrin clot covering the rupture site could be quite unstable, and any increase in blood pressure (with a corresponding increase in transmural pressure) could potentially dislodge this clot and allow rebleeding to occur. 26,27 Although the evidence is quite sparse and no randomized clinical trials (RCTs) currently exist, a meta-analysis (of case-controlled studies) from 2014 compared a systolic target of $140 \mathrm{~mm} \mathrm{Hg}$ to $160 \mathrm{~mm} \mathrm{Hg}$ and found that pressures only needed to be maintained below $160 \mathrm{~mm} \mathrm{Hg}$ to reduce the risk of rebleeding. ${ }^{27}$ Driving the blood pressure lower could potentially lead to cerebral ischemia. ${ }^{27}$ Until further definitive evidence emerges, maintaining the systolic blood pressure below $160 \mathrm{~mm} \mathrm{Hg}$ (or the mean arterial pressure below $100 \mathrm{~mm} \mathrm{Hg}$ ) is advisable. 
Once the aneurysm has been secured, it is appropriate to allow the patient's blood pressure to autoregulate up to a certain threshold. Although the exact value is unclear and there are patient specific considerations, a reasonable upper limit would be a systolic blood pressure around 180 to $200 \mathrm{~mm} \mathrm{Hg}{ }^{29}$

\section{Antifibrinolytic Therapy}

In the setting of aSAH, there are significant changes in both coagulation and fibrinolysis. ${ }^{26}$ Therapy directed at stabilizing the clot which covers the rupture site, by decreasing fibrinolysis, has shown promise. Antifibrinolytics, in particular tranexamic acid (TXA), significantly reduce aneurysm rebleeding rates, but unfortunately this has not led to an improvement in either mortality or functional outcomes. . $^{30,31}$

The major society guidelines offer contradictory recommendations. ${ }^{8,9}$ Further evidence from large, welldesigned RCTs is needed. Until then the decision to offer TXA therapy should be made on a case-by-case basis, administered for a short duration ( $<72$ hours) and used only if the delay in aneurysm securement is greater than 24 hours.

\section{Securing the Aneurysm: Endovascular Coiling versus Surgical Clipping}

To completely eliminate the risk of rebleeding, securing the aneurysm (via coiling or clipping) is the ultimate definitive therapy. ${ }^{32}$ The American Heart Association/American Stroke Association (AHA/ASA) guidelines recommend securing the aneurysm "... as early as feasible...." ${ }^{9}$ The European guidelines also recommend intervening as soon as possible but give a maximum time window of 72 hours from symptom onset. ${ }^{8}$

The International Subarachnoid Aneurysm Trial (ISAT) was a landmark trial. It remains the largest and most rigorous RCT to compare the endovascular coiling and surgical clipping approach to secure ruptured aneurysms. The endovascular group was more likely to have independent survival compared with the surgical group. Overall, the risk of late rebleeding in both groups was low but more frequent in the endovascular group. ${ }^{33,34}$ This trend continued up to 10 years after interventions. ${ }^{35}$ It is important to note that patients with posterior circulation, to a lesser extent middle cerebral artery aneurysms and poor-grade $\mathrm{SAH}$, were underrepresented in ISAT. The evidence on some aspects of coiling that remain unclear are (1) length of follow-up after aneurysm coiling and (2) the need for intervention when recanalization of the aneurysm neck after coil impaction. ${ }^{36}$

Endovascular intervention is a rapidly growing area. Standard coiling and balloon-assisted coiling are the indicated techniques for treatment of ruptured aneurysms. In this setting, stent-assisted coiling and flow diversion should be used only if the aneurysms are not treatable by conventional methods. This is in view of the need for antiplatelet therapy and limited evidence to support their use in emergency setting. ${ }^{37}$

\section{Vasospasm and Delayed Cerebral Ischemia}

Vasospasm is defined as radiographic narrowing of cerebral arteries, whereas delayed cerebral ischemia (DCI) is a clinical syndrome of focal neurological impairment. In aSAH, vasospasm is evident in $70 \%$ of patients, but only one-third of patients develop DCI. ${ }^{1}$ Typically, vasospasm starts 3 days after aneurysm rupture, peaks around days 7 to 10 , and resolves by day $21 .^{38}$ Until recently, the prevailing opinion was that cerebral vasospasm directly led to DCI. However, less than one-half of patients with vasospasm develop DCI, and the ischemic symptoms do not consistently correlate to the vascular territory affected by spasm. ${ }^{1}$ The process of $\mathrm{DCI}$ is not fully understood but is believed to occur at the cellular and microcirculatory levels, culminating in reduction in blood flow and tissue ischemia or infarction. ${ }^{39}$

\section{Monitoring and Diagnosis of Vasospasm or Delayed Cerebral Ischemia}

Monitoring of patients with poor-grade aSAH remains challenging. Clinical examination in those who are intubated and ventilated is often unreliable. There are three main modalities used to diagnose vasospasm, i.e., transcranial Doppler (TCD), CTA, and DSA. TCD is noninvasive and portable and demonstrates good correlation with DSA. However, it is operator dependent and reliant on the patient having good temporal acoustic windows and is an indirect measure of flow. ${ }^{40}$ The gold standard for diagnosis of vasospasm is DSA. The main disadvantages of DSA are that it is an invasive procedure that requires specialist skills and exposes the patient to significant contrast and radiation. ${ }^{40} \mathrm{CTA}$ has an excellent correlation with DSA in cases of severe vasospasm, less so in mild to moderate cases. ${ }^{41}$

CT perfusion (CTP) is another noninvasive imaging modality that is being used more frequently in recent years. The color maps of CTP provide information on cerebral blood volume, cerebral blood flow, and time parameters including mean transit time. At-risk territory demonstrates normal cerebral blood volume, diminished cerebral blood flow, and prolongation of mean transit time. ${ }^{42}$ CTP has the ability to demonstrate reversible ischemia, even in the absence of angiographic vasospasm. Compared with CTA, CTP is a better tool for diagnosing DCI in patients who are deteriorating clinically (-Fig. 3). ${ }^{43}$ Magnetic resonance imaging is another option to evaluate for ischemia: it is more sensitive than CTP study, but its use in critically ill patients is limited by logistical challenges. ${ }^{40}$

\section{Management of Vasospasm and Delayed Cerebral Ischemia}

Nimodipine is a calcium channel blocker and is considered a neuroprotectant in $\mathrm{aSAH} .{ }^{44}$ Both the American and European guidelines recommend giving nimodipine for a total of 21 days. $^{8,9}$ The exact mechanism by which nimodipine confers the observed benefits is unclear. It does not reduce the incidence or severity of vasospasm but is associated with reduction in the risk of both DCI and poor neurological outcome. ${ }^{1,45}$

Historically, when DCI was diagnosed, "triple H" therapy (hypertension, hypervolemia, hemodilution) was initiated to improve perfusion of the ischemic regions. However, it is associated with significant complications and the current 


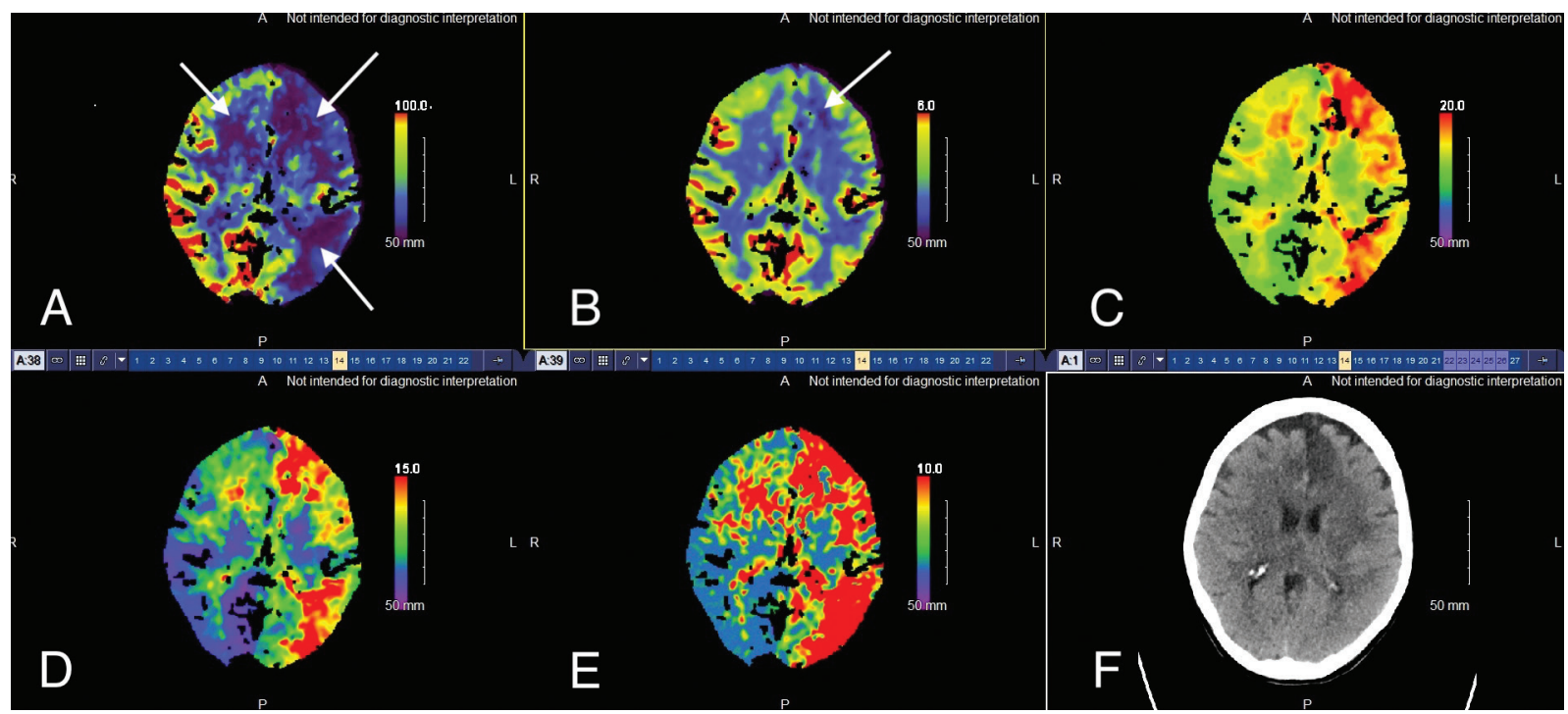

Fig. 3 Computed tomography perfusion. This study was performed day 10 post aSAH. Overall, this study is consistent with severe reversible ischemia in multiple territories. Panel A (cerebral blood flow) shows reduced blood flow in the bilateral anterior cerebral artery and left middle cerebral artery territories (white arrows). The flow reduction is most severe on the left side. Panel B (cerebral blood volume) shows a small area of reduced blood volume (white arrow) consistent with infarction. However, for the most part, cerebral blood volume is preserved. Panels C-E are time parameters that all show significant delays consistent with ischemia in the same regions as the flow reduction in panel $\mathbf{A}$. Panel $\mathrm{F}$ is a standard noncontrast CT head for comparison.

literature does not support its use..$^{29,46,47}$ Of the components, hypertensive therapy has the most supporting evidence. . $7,39,48,49^{-19}$ A recent retrospective observational study of 300 patients with DCI found that hypertensive therapy was associated with a reduction in both cerebral infarcts and poor clinical outcomes. ${ }^{39}$ In the induced hypertension group, $20 \%$ of patients developed infarcts compared with $33 \%$ in the group who did not receive therapy ${ }^{39}$ Unfortunately, an RCT that may have provided additional support was terminated early, due to slow recruitment and a lack of effect on cerebral perfusion. ${ }^{50}$

It is an accepted practice that if DCI fails to improve with blood pressure augmentation, trial of an inotropic drug may be acceptable. Some centers prefer the use of milrinone over dobutamine as it can be used as an intra-arterial injection, followed by intravenous infusion..$^{51}$ However, evidence from RCTs is lacking, and the use of milrinone may be associated with arterial hypotension, compromising hemodynamic stability, and, ultimately, perfusion to the brain. ${ }^{52}$

The absence of robust evidence and significant variation in practice is illustrated in major society guidelines, which make conflicting recommendations regarding hypertensive therapy for DCI: the AHA/ASA guidelines support its use whereas the European Stroke Organization guidelines do not. ${ }^{89}$

Based on the current literature and clinical experience, management strategies include the following:

1. Monitor for DCI with clinical examination (and possibly TCD). Perform a CTP study if there are concerns or the examination is unreliable.

2. To treat DCI, use blood pressure augmentation (induced hypertension) and escalate the blood pressure target in a stepwise fashion. If the symptoms improve, maintain that new target. Isotonic crystalloid (0.9\% saline) and vasopressors (norepinephrine, phenylephrine, or metaraminol) are used to achieve this goal.

3. If symptoms do not improve despite aggressive blood pressure augmentation (mean arterial pressure $>120 \mathrm{~mm} \mathrm{Hg}$ ) or induced hypertension is hampered by physiological decompensation, then intra-arterial intervention (angioplasty and vasodilators) could be considered, particularly if radiographic vasospasm is present and matches the area of deficit.

One final concern with induced hypertension is the possible rupture of coexisting, unsecured aneurysms. A retrospective review found no association between hypertensive therapy and rupture of these additional aneurysms..$^{53}$ As a result, induced hypertension should not be withheld in these patients.

\section{Endovascular Therapy}

Endovascular therapies including balloon angioplasty and selective intra-arterial vasodilator infusions, such as nimodipine, milrinone, and verapamil, have been used to treat vasospasm. Although the effectiveness has not been proven in RCTs, it may be a reasonable option in select groups of patients: those who are not improving despite conventional therapy or those with impaired cardiac function who are unable to cope with blood pressure augmentation. ${ }^{40,54}$

\section{Complications}

\section{Hydrocephalus}

Hydrocephalus is a common complication of aSAH, occurring in approximately 20 to $30 \%$ of patients..$^{55,56}$ The exact etiology 
is unclear, but it appears to occur due to both communicating (impaired absorption of CSF at arachnoid granulations) and noncommunicating (mechanical obstruction from clot or compression) components. ${ }^{55}$

A large proportion of cases of hydrocephalus identified on CT scan are not clinically significant and many resolve spontaneously. ${ }^{57}$ In a case series with 102 patients who developed acute hydrocephalus, only 31\% required CSF drainage. ${ }^{57}$ For patients who do require CSF drainage, placement of an external ventricular drain (EVD) is the usual treatment of choice. ${ }^{1}$ Indications for EVD placement include enlarged ventricles on CT and one of the following: (1) GCS 12 or less, (2) Hunt and Hess grade 2 or higher, (3) inability to follow commands, and (4) WFNS grade 2 or higher. ${ }^{12,58-60}$

The risks associated with EVD insertion include infection, hemorrhage, and aneurysm rebleeding. ${ }^{12}$ A meta-analysis from 2015 found the mean EVD infection prevalence to be $7.9 \%$ and the hemorrhage prevalence after EVD insertion to be $8.4 \%$ (0.7\% symptomatic). ${ }^{61}$ Regarding rebleeding, the current data are "conflicting" and no formal "causal relationship" has been established. ${ }^{58}$ Given the high morbidity and mortality associated with untreated acute hydrocephalus, these risks should not prevent the insertion of an EVD.

\section{Weaning from External Ventricular Drain and Shunt Dependence}

A significant percentage of patients with aSAH require permanent CSF diversion (shunt placement). A large retrospective cohort of 10,807 patients with aSAH showed that $6.5 \%$ ultimately required permanent CSF diversion. ${ }^{62}$

There are two main techniques to challenge the EVD. The first is to clamp the EVD and monitor for clinical deterioration or elevation in intracranial pressure. The second is a slow, progressive wean, increasing the drain height over several days while evaluating the clinical response..$^{32} \mathrm{~A}$ small RCT found that clamping the EVD led to shorter intensive care unit and hospital lengths of stay ${ }^{63}$ However, there were significant methodological concerns with the study, and further evidence is needed to definitively answer the question. ${ }^{64}$

\section{Seizures}

A systematic review from 2013 found that early seizures (during hospitalization) only occurred in $2.3 \%$ of patients with aSAH. The use of antiepileptic drugs did not reduce the incidence of early seizures and was associated with adverse events in over $20 \%$ of cases. ${ }^{65}$ Phenytoin, in particular, has been associated with multiple complications including fever, vasospasm, and worse cognitive outcomes at 3 months. ${ }^{65-67}$ Levetiracetam may lead to improved outcomes compared with phenytoin. ${ }^{32,65} \mathrm{At}$ this time, there is insufficient evidence to recommend routine seizure prophylaxis in aSAH.

\section{Fever}

Fever is a frequent complication of aSAH occurring in 40 to 90\% of patients. ${ }^{68-72}$ Approximately one-half of these cases are due to infection, whereas the remainder are labeled noninfectious. ${ }^{68,71}$ Neurogenic fever comprises the majority of this noninfectious category, but other causes (including medication, venous thromboembolic disease, and acalculous cholecystitis) need to be ruled out before this diagnosis can be made. ${ }^{71}$

Thermoregulation is controlled mainly by the hypothalamus, and either direct injury or irritation (from blood or inflammatory mediators in the CSF) can lead to a neurogenic fever. ${ }^{73}$ In $\mathrm{SAH}$, a higher clinical grade (more severe), a larger volume of subarachnoid blood, and the presence of intraventricular hemorrhage are all important risk factors for development of fever. ${ }^{73}$

Fever appears to be associated with worse outcomes in patients with aSAH. $68,69,72,74$

Management should include aggressive treatment of fever and maintenance of normothermia. Standard therapy consists of acetaminophen (paracetamol), nonsteroidal anti-inflammatory drugs, and surface cooling. ${ }^{36,69}$

\section{Cardiac Complications}

A variety of cardiac complications can occur in the setting of aSAH including dysrhythmias, ST-segment changes, elevated troponin, ventricular wall motion abnormalities, and myocardial stunning. ${ }^{36}$ Cardiac dysfunction, with reduction in ejection fraction or regional wall motion abnormalities, is present in 20 to $30 \%$ of patients with aSAH and is mainly driven by the sympathetic nervous system. ${ }^{75,76}$ Injury to the brain, in particular the insular cortex, leads to the triad of catecholamine release, autonomic dysfunction, and neuroinflammation. ${ }^{75}$ The end result of these processes on the myocardium is mitochondrial dysfunction and cell death. ${ }^{75}$ This occurs in a pattern that corresponds to areas of sympathetic innervation (e.g., subendocardial regions with apical sparing) as opposed to a specific coronary artery vascular territory. ${ }^{75}$

This so-called neurogenic stunned myocardium typically occurs within 48 hours of onset of aSAH and resolves within 2 weeks. ${ }^{76}$ Some patients will be asymptomatic, whereas others will develop cardiogenic shock and pulmonary edema. It is important to closely follow cardiac biomarkers and serial electrocardiograms to monitor for evidence of territorial ischemia that could require cardiac catheterization. Supportive care consists of optimizing cardiac output, when necessary, with medications or mechanical supports. ${ }^{76}$

Given the significant role of endogenous catecholamines, combined $\alpha$ - and $\beta$-blockade would seem like a reasonable treatment. There is limited evidence from a small RCT that using propranolol and phentolamine may improve neurological outcomes, but it did not have an impact on mortality. ${ }^{76}$ Until more robust clinical data exist, this approach cannot be recommended.

\section{Electrolyte Abnormalities Hyponatremia}

In aSAH, hyponatremia $(\mathrm{Na}<135 \mathrm{mmol} / \mathrm{L})$ is the most common disorder of sodium, occurring in up to $40 \%$ of patients. ${ }^{29}$ Seizures and cerebral edema can occur when the sodium drops rapidly or if severe hyponatremia develops. ${ }^{29}$ The two main causes are cerebral salt wasting (CSW) and syndrome of inappropriate antidiuretic hormone (SIADH). ${ }^{29,36}$ Distinguishing between them can be challenging; however, 
polyuria and volume depletion are usually features of CSW (although not $100 \%$ confirmatory)..$^{29,36,77}$

Classically, the treatment for SIADH would be fluid restriction. ${ }^{77}$ However, in the setting of aSAH, fluid restriction can lead to hypovolemia and decreased cardiac output, which in turn can worsen DCI and, ultimately, impact outcomes. ${ }^{29,77}$ As a result, treatment for both conditions should be the same: maintenance of euvolemia and sodium replacement (either hypertonic saline infusion or enteral salt administration).29,77 Overly rapid correction ( $>1 \mathrm{mmol} / \mathrm{h}$ or $>12 \mathrm{mmol} / 24 \mathrm{~h}$ ) can lead to central pontine myelinolysis so frequent monitoring is important. ${ }^{36}$ Commencement of fludrocortisone can be helpful in maintaining a normal serum sodium and should be considered as adjunctive therapy. ${ }^{29,77,78}$

\section{Hypernatremia}

Hypernatremia is much less common than hyponatremia in aSAH and usually occurs due to inadequate free water replacement, volume loss, or excess sodium administration. ${ }^{36,49}$ Central diabetes insipidus (DI) can occur from hypothalamic dysfunction but is very infrequent in SAH. ${ }^{36,49}$ Maintaining fluid balance can be a challenge in managing patients with $\mathrm{DI}$, as significant prolonged polyuria is often a key feature.

\section{Conclusion}

Prevention of secondary brain injury is the fundamental goal in the management of aSAH. Sophisticated monitoring and interventional techniques have become more accessible in recent years. It remains prudent to ensure that meticulous care is provided.

\section{Conflict of interest}

None declared.

\section{References}

1 Lawton MT, Vates GE. Subarachnoid hemorrhage. N Engl J Med 2017;377(3):257-266

2 Degen LAR, Dorhout Mees SM, Algra A, Rinkel GJE. Interobserver variability of grading scales for aneurysmal subarachnoid hemorrhage. Stroke 2011;42(6):1546-1549

3 Rivero-Arias O, Gray A, Wolstenholme J. Burden of disease and costs of aneurysmal subarachnoid haemorrhage (aSAH) in the United Kingdom. Cost Eff Resour Alloc 2010;8:6

4 Taufique Z, May T, Meyers E, et al. Predictors of poor quality of life 1 year after subarachnoid hemorrhage. Neurosurgery 2016;78(2):256-264

5 Al-Khindi T, Macdonald RL, Schweizer TA. Cognitive and functional outcome after aneurysmal subarachnoid hemorrhage. Stroke 2010;41(8):e519-e536

6 Huhtakangas J, Lehto H, Seppä K, et al. Long-term excess mortality after aneurysmal subarachnoid hemorrhage: patients with multiple aneurysms at risk. Stroke 2015;46(7): $1813-1818$

7 Rinkel GJ, Djibuti M, Algra AA, van Gijn J. Prevalence and risk of rupture of intracranial aneurysms: a systematic review. Stroke 1998;29(1):251-256

8 Steiner T, Juvela S, Unterberg A, Jung C, Forsting $M$, Rinkel G; European Stroke Organization. European Stroke
Organization guidelines for the management of intracranial aneurysms and subarachnoid haemorrhage. Cerebrovasc Dis 2013;35(2):93-112

9 Connolly ES Jr, Rabinstein AA, Carhuapoma JR, et al; American Heart Association Stroke Council; Council on Cardiovascular Radiology and Intervention; Council on Cardiovascular Nursing; Council on Cardiovascular Surgery and Anesthesia; Council on Clinical Cardiology. Guidelines for the management of aneurysmal subarachnoid hemorrhage: a guideline for healthcare professionals from the American Heart Association/ American Stroke Association. Stroke 2012;43(6):1711-1737

10 Kataoka K, Taneda M, Asai T, Kinoshita A, Ito M, Kuroda R. Structural fragility and inflammatory response of ruptured cerebral aneurysms. A comparative study between ruptured and unruptured cerebral aneurysms. Stroke 1999;30(7): 1396-1401

11 Zheng VZ, Wong GKC. Neuroinflammation responses after subarachnoid hemorrhage: a review. J Clin Neurosci 2017;42:7-11

12 Macdonald RL, Schweizer TA. Spontaneous subarachnoid haemorrhage. Lancet 2017;389(10069):655-666

13 Bassi P, Bandera R, Loiero M, Tognoni G, Mangoni A. Warning signs in subarachnoid hemorrhage: a cooperative study. Acta Neurol Scand 1991;84(4):277-281

14 Perry JJ, Stiell IG, Sivilotti MLA, et al. Sensitivity of computed tomography performed within six hours of onset of headache for diagnosis of subarachnoid haemorrhage: prospective cohort study. BMJ 2011;343:d4277

15 Cortnum S, Sørensen P, Jørgensen J. Determining the sensitivity of computed tomography scanning in early detection of subarachnoid hemorrhage.Neurosurgery2010;66(5):900-902, discussion 903

16 van der Wee N, Rinkel GJ, Hasan D, van Gijn J. Detection of subarachnoid haemorrhage on early CT: is lumbar puncture still needed after a negative scan? J Neurol Neurosurg Psychiatry 1995;58(3):357-359

17 Agid R, Andersson T, Almqvist H, et al. Negative CT angiography findings in patients with spontaneous subarachnoid hemorrhage: when is digital subtraction angiography still needed? AJNR Am J Neuroradiol 2010;31(4):696-705

18 Takagi K, Tamura A, Nakagomi $T$, et al. How should a subarachnoid hemorrhage grading scale be determined? A combinatorial approach based solely on the Glasgow coma scale. J Neurosurg 1999;90(4):680-687

19 Report of World Federation of Neurological Surgeons Committee on a Universal Subarachnoid Hemorrhage Grading Scale. J Neurosurg 1988;68(6):985-986

20 Hunt WE, Hess RM. Surgical risk as related to time of intervention in the repair of intracranial aneurysms. J Neurosurg 1968;28(1):14-20

21 van Heuven AW, Dorhout Mees SM, Algra A, Rinkel GJE. Validation of a prognostic subarachnoid hemorrhage grading scale derived directly from the Glasgow coma scale. Stroke 2008;39(4):1347-1348

22 Lindsay KW, Teasdale G, Knill-Jones RP, Murray L. Observer variability in grading patients with subarachnoid hemorrhage. J Neurosurg 1982;56(5):628-633

23 Fisher CM, Kistler JP, Davis JM. Relation of cerebral vasospasm to subarachnoid hemorrhage visualized by computerized tomographic scanning. Neurosurgery 1980;6(1):1-9

24 Frontera JA, Claassen J, Schmidt JM, et al. Prediction of symptomatic vasospasm after subarachnoid hemorrhage: the modified Fisher scale. Neurosurgery 2006;59(1):21-27, discussion 21-27

25 Kramer AH, Hehir M, Nathan B, et al. A comparison of 3 radiographic scales for the prediction of delayed ischemia and 
prognosis following subarachnoid hemorrhage. J Neurosurg 2008;109(2):199-207

26 Larsen CC, Astrup J. Rebleeding after aneurysmal subarachnoid hemorrhage: a literature review. World Neurosurg 2013;79(2):307-312

27 Tang CC, Zhang TS, Zhou LF. Risk factors for rebleeding of aneurysmal subarachnoid hemorrhage: a meta-analysis. PLoS One 2014;9(6):e99536

28 Darkwah Oppong M, Gümüs M, Pierscianek D, et al. Aneurysm rebleeding before therapy: a predictable disaster? J Neurosurg 2018. doi: 10.3171/2018.7.JNS181119

29 Datar S, Rabinstein AA. Postinterventional critical care management of aneurysmal subarachnoid hemorrhage. Curr Opin Crit Care 2017;23(2):87-93

30 Baharoglu MI, Germans MR, Rinkel GJE, et al. Antifibrinolytic therapy for aneurysmal subarachnoid haemorrhage. Cochrane Database Syst Rev 2013;(8):CD001245

31 Anker-Møller T, Troldborg A, Sunde N, Hvas AM. Evidence for the use of tranexamic acid in subarachnoid and subdural hemorrhage: a systematic review. Semin Thromb Hemost 2017;43(7):750-758

32 Rabinstein AA, Lanzino G. Aneurysmal Subarachnoid hemorrhage: unanswered questions. Neurosurg Clin N Am 2018;29(2):255-262

33 Molyneux A, Kerr R, Stratton I, et al; International Subarachnoid Aneurysm Trial (ISAT) Collaborative Group. International Subarachnoid Aneurysm Trial (ISAT) of neurosurgical clipping versus endovascular coiling in 2143 patients with ruptured intracranial aneurysms: a randomised trial. Lancet 2002; 360(9342):1267-1274

34 Molyneux AJ, Kerr RSC, Yu LM, et al; International Subarachnoid Aneurysm Trial (ISAT) Collaborative Group. International Subarachnoid Aneurysm Trial (ISAT) of neurosurgical clipping versus endovascular coiling in 2143 patients with ruptured intracranial aneurysms: a randomised comparison of effects on survival, dependency, seizures, rebleeding, subgroups, and aneurysm occlusion. Lancet 2005;366(9488):809-817

35 Molyneux AJ, Birks J, Clarke A, Sneade M, Kerr RSC. The durability of endovascular coiling versus neurosurgical clipping of ruptured cerebral aneurysms: 18 year follow-up of the UK cohort of the International Subarachnoid Aneurysm Trial (ISAT) Lancet 2015;385(9969):691-697

36 Hall A, O'Kane R. The extracranial consequences of subarachnoid hemorrhage. World Neurosurg 2018;109:381-392

37 Pierot L, Wakhloo AK. Endovascular treatment of intracranial aneurysms: current status. Stroke 2013;44(7):2046-2054

38 Dorsch NW, King MT. A review of cerebral vasospasm in aneurysmal subarachnoid haemorrhage part I: Incidence and effects. J Clin Neurosci 1994;1(1):19-26

39 Haegens NM, Gathier CS, Horn J, Coert BA, Verbaan D, van den Bergh WM. Induced hypertension in preventing cerebral infarction in delayed cerebral ischemia after subarachnoid hemorrhage. Stroke 2018;49(11):2630-2636

40 Rabinstein AA, Lanzino G, Wijdicks EF. Multidisciplinary management and emerging therapeutic strategies in aneurysmal subarachnoid haemorrhage. Lancet Neurol 2010;9(5): 504-519

41 Anderson GB, Ashforth R, Steinke DE, Findlay JM. CT angiography for the detection of cerebral vasospasm in patients with acute subarachnoid hemorrhage. Am J Neuroradiol 2000;21(6):1011-1015

42 Lui YW, Tang ER, Allmendinger AM, Spektor V. Evaluation of CT perfusion in the setting of cerebral ischemia: patterns and pitfalls. AJNR Am J Neuroradiol 2010;31(9):1552-1563

43 Dankbaar JW, de Rooij NK, Velthuis BK, Frijns CJM, Rinkel GJE, van der Schaaf IC. Diagnosing delayed cerebral ischemia with different CT modalities in patients with subarachnoid hemorrhage with clinical deterioration. Stroke 2009;40(11):3493-3498

44 Pickard JD, Murray GD, Illingworth R, et al. Oral nimodipine and cerebral ischaemia following subarachnoid haemorrhage. Br J Clin Pract 1990;44(2):66-67

45 Dorhout Mees SM, Rinkel GJE, Feigin VL, et al. Calcium antagonists for aneurysmal subarachnoid haemorrhage. Cochrane Database Syst Rev 2007;(3):CD000277

46 Loan JJM, Wiggins AN, Brennan PM. Medically induced hypertension, hypervolaemia and haemodilution for the treatment and prophylaxis of vasospasm following aneurysmal subarachnoid haemorrhage: systematic review. $\mathrm{Br} J$ Neurosurg 2018;32(2):157-164

47 Tagami T, Kuwamoto K, Watanabe A, et al; SAH PiCCO Study Group. Effect of triple-H prophylaxis on global end-diastolic volume and clinical outcomes in patients with aneurysmal subarachnoid hemorrhage. Neurocrit Care 2014;21(3):462-469

48 Dankbaar JW, Slooter AJ, Rinkel GJ, Schaaf IC. Effect of different components of triple-H therapy on cerebral perfusion in patients with aneurysmal subarachnoid haemorrhage: a systematic review. Crit Care 2010;14(1):R23

49 Okazaki T, Kuroda Y. Aneurysmal subarachnoid hemorrhage: intensive care for improving neurological outcome. J Intensive Care 2018;6(1):28

50 Gathier CS, van den Bergh WM, van der Jagt M, et al; HIMALAIA Study Group. Induced hypertension for delayed cerebral ischemia after aneurysmal subarachnoid hemorrhage: a randomized clinical trial. Stroke 2018;49(1):76-83

51 Diringer MN, Bleck TP, Claude Hemphill J III, et al; Neurocritical Care Society. Critical care management of patients following aneurysmal subarachnoid hemorrhage: recommendations from the Neurocritical Care Society's Multidisciplinary Consensus Conference. Neurocrit Care 2011;15(2):211-240

52 Baumann A, Derelle A-L, Mertes P-M, Audibert G. Seeking new approaches: milrinone in the treatment of cerebral vasospasm. Neurocrit Care 2012;16(3):351-353

53 Reynolds MR, Buckley RT, Indrakanti SS, et al. The safety of vasopressor-induced hypertension in subarachnoid hemorrhage patients with coexisting unruptured, unprotected intracranial aneurysms. J Neurosurg 2015;123(4):862-871

54 Adami D, Berkefeld J, Platz J, et al. Complication rate of intraarterial treatment of severe cerebral vasospasm after subarachnoid hemorrhage with nimodipine and percutaneous transluminal balloon angioplasty: worth the risk? J Neuroradiol 2019;46(1):15-24

55 Germanwala AV, Huang J, Tamargo RJ. Hydrocephalus after aneurysmal subarachnoid hemorrhage. Neurosurg Clin N Am 2010;21(2):263-270

56 Lu J, Ji N, Yang Z, Zhao X. Prognosis and treatment of acute hydrocephalus following aneurysmal subarachnoid haemorrhage. J Clin Neurosci 2012;19(5):669-672

57 Hasan D, Vermeulen M, Wijdicks EF, Hijdra A, van Gijn J. Management problems in acute hydrocephalus after subarachnoid hemorrhage. Stroke 1989;20(6):747-753

58 Gigante P, Hwang BY, Appelboom G, Kellner CP, Kellner MA, Connolly ES. External ventricular drainage following aneurysmal subarachnoid haemorrhage. $\mathrm{Br} \mathrm{J}$ Neurosurg 2010;24(6):625-632

59 Dorai Z, Hynan LS, Kopitnik TA, Samson D. Factors related to hydrocephalus after aneurysmal subarachnoid hemorrhage. Neurosurgery 2003;52(4):763-769, discussion 769-771

60 Corsten L, Raja A, Guppy K, et al. Contemporary management of subarachnoid hemorrhage and vasospas: the UIC experience. Surg Neurol 2001;56(3):140-148, discussion 148-150

61 Dey M, Stadnik A, Riad F, et al; CLEAR III Trial Investigators. Bleeding and infection with external ventricular drainage: a 
systematic review in comparison with adjudicated adverse events in the ongoing Clot Lysis Evaluating Accelerated Resolution of Intraventricular Hemorrhage Phase III (CLEAR-III IHV) trial. Neurosurgery 2015;76(3):291-300, discussion 301

62 Lai L, Morgan MK. Predictors of in-hospital shunt-dependent hydrocephalus following rupture of cerebral aneurysms. J Clin Neurosci 2013;20(8):1134-1138

63 Klopfenstein JD, Kim LJ, Feiz-Erfan I, et al. Comparison of rapid and gradual weaning from external ventricular drainage in patients with aneurysmal subarachnoid hemorrhage: a prospective randomized trial. J Neurosurg 2004;100(2):225-229

64 Chung DY, Mayer SA, Rordorf GA. External ventricular drains after subarachnoid hemorrhage: is less more? Neurocrit Care 2018;28(2):157-161

65 Raper DMS, Starke RM, Komotar RJ, Allan R, Connolly ES Jr. Seizures after aneurysmal subarachnoid hemorrhage: a systematic review of outcomes. World Neurosurg 2013;79(5-6):682-690

66 Naidech AM, Kreiter KT, Janjua N, et al. Phenytoin exposure is associated with functional and cognitive disability after subarachnoid hemorrhage. Stroke 2005;36(3):583-587

67 Rosengart AJ, Huo JD, Tolentino J, et al. Outcome in patients with subarachnoid hemorrhage treated with antiepileptic drugs. J Neurosurg 2007;107(2):253-260

68 Kramer CL, Pegoli M, Mandrekar J, Lanzino G, Rabinstein AA. Refining the association of fever with functional outcome in aneurysmal subarachnoid hemorrhage. Neurocrit Care 2017;26(1):41-47

69 Scaravilli V, Tinchero G, Citerio G; Participants in the International Multi-Disciplinary Consensus Conference on the
Critical Care Management of Subarachnoid Hemorrhage. Fever management in SAH. Neurocrit Care 2011;15(2):287-294

70 Lai PMR, See AP, Silva MA, et al. Non-infectious fever in aneurysmal subarachnoid hemorrhage: association with cerebral vasospasm and clinical outcome. World Neurosurg 2018;1-23

71 Meier K, Lee K. Neurogenic fever. J Intensive Care Med 2017;32(2):124-129

72 Fernandez A, Schmidt JM, Claassen J, et al. Fever after subarachnoid hemorrhage: risk factors and impact on outcome. Neurology 2007;68(13):1013-1019

73 Badjatia N. Hyperthermia and fever control in brain injury. Crit Care Med 2009;37(7, Suppl):S250-S257

74 Naidech AM, Bendok BR, Bernstein RA, et al. Fever burden and functional recovery after subarachnoid hemorrhage. Neurosurgery 2008;63(2):212-217, discussion 217-218

75 Krishnamoorthy V, Mackensen GB, Gibbons EF, Vavilala MS. Cardiac dysfunction after neurologic injury: what do we know and where are we going? Chest 2016;149(5):1325-1331

76 Murthy SB, Shah S, Rao CPV, Bershad EM, Suarez JI. Neurogenic stunned myocardium following acute subarachnoid hemorrhage: pathophysiology and practical considerations. J Intensive Care Med 2015;30(6):318-325

77 Garg R, Bar B. Systemic complications following aneurysmal subarachnoid hemorrhage. Curr Neurol Neurosci Rep 2017; 17(1):7

78 Raya AK, Diringer MN. Treatment of subarachnoid hemorrhage. Crit Care Clin 2014;30(4):719-733 